\title{
Diagnosis of diseases using data mining
}

\section{Type of article: conference abstract}

\author{
Gozali Elahe1, Dr Rahimi Bahlol2, Sadeghi Malihe3, Dr Safdari Reza4* \\ 1: PhD student of Health Information Management, Department of Health Information \\ Management, School of Allied Medical Sciences, Tehran University of Medical Sciences \\ (TUMS), Tehran, Iran \\ 2: Department of health information management, Urmia University of Medical \\ Science, Urmia, Iran \\ 3: Department of health information management, Iran University of Medical Science, \\ Tehran, Iran \\ 4: Department of health information management, School of Allied Medical Sciences, \\ Tehran University of Medical Sciences (TUMS), Tehran, Iran \\ *Tel: 02188983025 E-mail: rsafdari@tums.ac.ir
}

\begin{abstract}
Introduction: In the information age, data are the most important asset for health organizations. In the case of using data in useful and optimal manner, they can become financial resources for organization. Data mining is an appropriate method to transform this potential value into strategic information. Data mining means extraction of hidden information, recognition of hidden relationships and patterns, and in general, discovery of useful knowledge at high volume. The objective of this review paper was to evaluate using data mining in diagnoses of diseases.

Methods: This research is a review paper conducted based on a structured review of the papers published in Science Direct, Pubmed, Google Scholar, SID, Magiran (between years 2005 and 2015) and books related to using data mining in medical science and using it in diagnose of diseases with related keywords.

Results: Nowadays, data mining is used in many medical science studies, including diagnosis of diseases, discovering the hidden patterns in data, and so on. New ideas such as discovery of Knowledge from Discovery and Data Mining Database, which includes data mining techniques, have found more popularity and they has becomedesired research tool for researchers. Researchers can use them to identify patterns and relationshipsamong great number of variables. Using them, researchers have been able to predict theresults obtained from one disease by using information stores available in databases.

Several studies have indicated that data mining is used widely in diagnosis of diseases based on types of information (medical images, characteristics of patients, and so on), such as tuberculosis, types of cancers, infectious diseases, and diagnosis of anomalies rarely diagnosed by human (spots and particular points within aye, which is the symptom of onset of blindness resulting from diabetes), determining type of behavior with patients, and predicting the success rate of surgical surgeries, determining the success rate of therapeutic methods in coping with incurable diseases, and so on.
\end{abstract}

Conclusion: One of the most important challenging topics in healthcare is transformation of raw clinical data into meaningful information following continuous generation of great number of data. In current competitive environment, health organizations using technologies such as data mining to improve healthcare quality will achieve success faster. Many of research centers in Iran are faced with large volume of information, which is not analyzed at all or will be time-consuming due to using traditional methods, even in the case of using analysis and converting them to knowledge. In light of using data mining and its implementation, health organizations can transform the data into a powerful and competitive tool and take new steps in preventing, diagnosing, treating, and providing high-quality services for clients.

KEYWORDS: Data mining, diagnosis of diseases, healthcare organizations

\section{Declaration of conflicts}

This abstract is selected from the First International Congress of Diseases and Health Outcomes Registry and First National Congress of Medical Informatics, 14-17 February 2017, Mashhad, Iran 
Medical Technologies Journal, Volume: 1, Issue: 4, October-December 2017, Pages:76-136. Doi : https://doi.org/10.26415/2572-004X-vol1iss4

\section{Authors' biography}

No biography.

\section{References}

No references. 